ARTÍCULO ORIGINAL

\title{
INFORMALIDAD EN LAS MYPES Y SU INFLUENCIAEN LA RECAUDACIÓN DEL IMPUESTO GENERAL A LAS VENTAS
}

\section{INFORMALITY IN THE MYPES AND ITS INFLUENCE IN THE COLLECTION OF THE GENERAL SALES TAX}

\author{
Dany Mostacero Mayo \\ Universidad Nacional Mayor de San Marcos \\ Egresado de la Maestria en Politica y sistema tributario- UNMSM. \\ Correo : danymostacero@hotmail.com \\ [Recibido: 29/08/2017 Aceptado: 15/04/2018]
}

\begin{abstract}
RESUMEN
La investigación permitió explicar la Existencia de influencia de la Dimensión de la Informalidad de las Mypes en la Recaudación del IGV, durante el periodo 2010-2014, y por qué la Informalidad de las Mypes fue un factor que influencio en la Recaudación del IGV. Periodo 2010-2014, A partir de dicho objetivo, se realizó un análisis descriptivo - explicativo sobre la relación entre las variables para esto, se utilizó data oficial del Ministerio de la Producción, ministerio de trabajo, sunat entre otros.Siendo así, se obtuvo como principal resultado que si existe Influencia de la Dimensión de la Informalidad de las MYPES en la Recaudación del IGV en el periodo 2010-2014 y que el grado de dicha Influencia es estadísticamente significativa. De este modo, se concluyó que la Informalidad presente en las Mypes, afecta significativamente la recaudación del IGV. Y consiguientemente, los ingresos tributarios del gobierno central y es la principal barrera para lograr la tan necesaria ampliación de la base Tributaria. Además, La Informalidad es uno de los problemas que el gobierno no ha podido dar solución, las cifras señalan que estas, inclusive se encuentran por encima de las empresas que operan respetando las normas internas. Y si bien este tipo de empresas crean escenarios favorables para la captación de mano de obra, generan distorsiones severas que en nada contribuyen al proceso de desarrollo.
\end{abstract}

Palabras claves : Micro y Pequeña Empresa, Informalidad, Base tributaria, Evasión, Recaudación.

\begin{abstract}
The investigation explains the existence of influence of the informality dimension of the MYPES in the collection of the VAT, during the period 2010-2014, and why the informality of the Mypes was a factor that influenced the collection of VAT. In the period 2010-2014, based on this objective, a descriptive - explanatory analysis was carried out on the relationship between the variables, using official data from the Ministry of Production, Ministry of Labor, Sunat, among others. The main result shows that there is an Influence of the informality dimension of the MYPES in the Collection of the IGV in the period 2010-2014 and that the degree of this influence is statistically significant. In this way, it was concluded that the informality presented in the Mypes, significantly affects the collection of the VAT. And consequently, the tax revenues of the central government and is the main barrier to achieve the much-needed expansion of the tax base. In addition, informality is one of the problems that the government has not been able to solve, the figures indicate that these are even above the companies that operate in compliance with internal regulations. And while these types of companies create favorable scenarios for attracting labor, they generate severe distortions that contribute nothing to the development process.
\end{abstract}

Keywords: Micro and Small Business, Informality, Tax Base, Evasion, Collection.

\section{INTRODUCCIÓN}

El estudio examina la relación entre la informalidad en las Mypes y la Evasión del IGV, por lo que se formula la siguiente interrogante: ¿Cuál es la dimensión de la Informalidad de las Mypes en la Recaudación Fiscal? Es así que la hipótesis que responde a la pregunta es: "La dimensión de la informalidad de las Mypes afecta la recaudación fiscal".
Para tal efecto, se exponen los fundamentos de La Teoría Estructuralista del Sector Informal (José I. Uribe et al. 2006), también se exponen los fundamentos de La Teoría de los Mercados Internos de Trabajo (José I. 
Uribe et al. (2006), y de la Teoría Institucionalista del Sector Informal (José I. Uribe et al. (2006) sostiene que un "un aspecto importante en el presente estudio, es el análisis de los datos que estiman el número total de Mypes en el Perú; De las Mipymes en Cifras 2014, publicado por el Ministerio de la Producción; Y de los Ingresos del Gobierno Central consolidado 2015, publicados por la sunat".

Bajo esta premisa, el estudio recurre al enfoque descriptivo, porque se estudian indicadores que permitirán determinar la influencia de la informalidad de la Mypes en la recaudación del IGV. Por cierto, la principal limitación de la investigación es que, a la fecha la evasión tributaria se calcula en base a datos aproximados ya que no aparece información estadística oficial de las cuentas nacionales.

En lo esencial este estudio, analiza la realidad de las Mypes, que representan un papel muy importante en la economía, no obstante el terreno donde se desarrollan todavía es muy frágil, debido a sus bajos índices de supervivencia y consolidación empresarial, y esto debido a que hay un fenómeno estrechamente vinculado a las Mypes: La informalidad, que según Gomero (2015) dice: "La Informalidad es uno de los problemas que el gobierno no ha podido dar solución, las cifras señalan que estas, inclusive se encuentran por encima de las empresas que operan respetando las normas internas. Si bien este tipo de empresas crean escenarios favorables para la captación de mano de obra, pero generan distorsiones severas que en nada contribuyen al proceso de desarrollo"(p.30)

Uribe, Ortiz y Castro; (2006) desarrollaron tres teorías sobre la informalidad las que nos muestran dife- rentes puntos de vista sobre el origen, causa y efectos de la informalidad; Que según: Economía Y Desarrollo, Articulo: Una teoría general sobre la informalidad laboral, 5(2).

Uribe et al. (2006), dice: "Una larga tradición teórica de corte estructuralista ha pensado la informalidad laboral como el resultado de un escaso desarrollo del sector moderno de la economía, de tal manera que éste no alcanza a absorber toda la fuerza laboral disponible. La población excedente, sea educada o no, se ve forzada a laborar en actividades informales de baja remuneración o cae en el desempleo.

Lo más característico de la visión estructuralista es el dualismo económico; el sector moderno comprende el conjunto de actividades económicas en las cuales existen economías de escala en capital físico y capital humano; por otra parte, el sector tradicional o informal se caracteriza por escasos requerimientos de capital físico y capacitación laboral. La baja productividad del sector informal se explica por sobredimensionamiento laboral:

- Dados los bajos costos de entrada en el sector, no es posible contener la entrada de trabajadores por encima de los niveles de eficiencia.

- Por tanto, el sector informal tiende a operar en unidades pequeñas con baja productividad.

- Como resultado surgen desigualdades significativas entre los trabajadores que se enganchan en el sector moderno y aquellos que no lo logran.

Además de recibir un menor ingreso percápita, los informales tienen empleos de menor calidad pues las empresas informales que generan es- casas rentas; Tienden a incumplir las regulaciones institucionales y legales. En consecuencia, los empleos informales se caracterizan por: inestabilidad laboral, ausencia de prestaciones sociales, ausencia de contrato laboral, condiciones de trabajo inseguro y antihigiénico.

De acuerdo con la teoría estructuralista, el sector informal es el producto de la falta de correspondencia, tanto cuantitativa como cualitativa, entre la demanda y la oferta de trabajo, la cual, a su vez, es el resultado de la forma en que la estructura económica incide en el mercado laboral. (...), Además de que existen diferencias significativas entre los sectores formal e informal, éstas tienden a acentuarse en el tiempo. Dicho de otro modo, las características de uno y otro sector tienden a autorreforzarse.

Dada una cierta complementariedad entre capital físico y capital humano, es fácil entender que aquellos sectores modernos que utilizan tecnologías más avanzadas requieren trabajadores más calificados y ésta, a su vez, es otra barrera de entrada a la actividad formal porque típicamente los países subdesarrollados cuentan con una escasa fuerza laboral calificada”.

(José I. Uribe et al. 2006), dice: “Una vertiente estructuralista ha hecho énfasis en la existencia de Mercados Internos de Trabajo (MIT) en el sector moderno. Para ella, las asociaciones de empleados, los sindicatos, y las convenciones colectivas regulan la contratación y el ascenso laboral, de tal manera que terminan presionando las remuneraciones laborales hacia arriba (HARRIS y TODARO, 1970; DOERINGER y PIORE, 1971, 1975; PIORE, 1975, 1980; TAUBMAN y WACHTER, 1986; TOKMAN y KLEIN, 1996). 
Por otra parte, el mercado de trabajo en el sector tradicional o informal es más competitivo pues no está sujeto a las regulaciones de los mercados internos de trabajo Bourguignon, (1979). Así, pues, en esta visión la economía también se caracteriza por mercados de trabajo segmentados: dada la existencia de mercados internos de trabajo en el sector moderno, el flujo de trabajo del sector informal al formal está fuertemente restringido. Se puede afirmar que la visión MIT presupone la estructuralista, pues ambas aceptan la existencia de dualismo productivo.

Como los estructuralistas, los teóricos de la visión MIT plantean que el dualismo tiene un fundamento tecnológico en la existencia de economías a escala (PIORE, 1975, 1980). También aportan el análisis de las instituciones laborales que se construyen en el sector moderno de la economía. Según esta visión, en los sectores modernos surgen formas específicas de regulación que se denominan corrientemente mercados internos de trabajo:

- Empresa u otra unidad administrativa que se caracteriza por tener escalas de puestos de trabajo. Éstos están protegidos, a excepción de los del puerto de entrada, de las presiones competitivas del mercado, en el sentido de que los salarios y el empleo se rigen por reglas y procedimientos administrativos y no son determinados por las fuerzas de la oferta y la demanda. (MCCONNELL y BRUE, 1997, p. 537).

Existen varias razones esgrimidas en la literatura para entender por qué las empresas acceden a la formación de mercados internos: tener un interlocutor representativo, mantener la paz laboral, preservar a los mejores traba- jadores, generar un clima de trabajo que promueva la eficiencia y el compromiso de largo plazo de los trabajadores con la empresa, disminuir la rotación del personal y los costos de entrenamiento, entre otros. Pero el costo que deben pagar las empresas por esos beneficios implícitos es la limitación de la oferta de trabajo a los internos (por oposición a los trabajadores externos), lo cual les da poder de negociación a los trabajadores asociados (MCCONNELL y BRUE, 1997, cap. 14).(...), La literatura asociada con la visión MIT se caracteriza por su incredulidad sobre las principales premisas que soportan el modelo neoclásico: existencia de un mercado competitivo, comportamiento racional de los agentes (optimización), salarios flexibles, ajuste en el volumen de empleo generado de acuerdo con los cambios en los salarios, movilidad intersectorial, etc."

(José I. Uribe et al. 2006), dice: “Se la denomina teoría institucionalista porque se centra en los costos que el Estado y en general las instituciones le imponen al funcionamiento, legalización y desempeño de las empresas. Esta visión aboga en cierta forma por un libre funcionamiento de los mercados y plantea de forma explícita o implícita una desconfianza en la intervención estatal en la economía.

Como es bien conocido, en el contexto de un Estado de Derecho existen regulaciones para el funcionamiento de las empresas, y existen además obligaciones de diferente tipo: tributarias (impuestos a la renta, a las ventas, de industria y comercio, prediales, etc.), laborales (salario mínimo, pensiones, cesantías, parafiscales), pagos de servicios públicos de actividad económica, sanitarias, ambientales, etc.

En este argumento es muy importan- te el grado de ineficiencia y corrupción de los gobiernos, pues este factor alarga el proceso de legalización y los costos relacionados con ese proceso. En general, tanto la ineficiencia del Estado como los costos de sus servicios y regulaciones se pueden entender como costos de transacción que desalientan la formalización de las empresas.

Por otra parte, De Soto (1987), dice: "la posibilidad de endeudarse para invertir está usualmente restringida para aquellos sectores de la población que no cuentan con patrimonio o cuyo patrimonio no está legalizado".

Asimismo Soto (1987) sostiene que "esta situación configura una barrera a la entrada en la formalidad porque las empresas permanecen por fuera del marco institucional ante la incapacidad de convertir sus activos en capital líquido para invertir. El enfoque institucionalista ignora las restricciones que puedan provenir de la estructura económica y se concentra en las decisiones que los individuos toman con respecto a la participación en la actividad económica. Por ello plantean que los agentes toman sus decisiones con base en un análisis costo beneficio cuyas opciones son pertenecer al sector formal o al informal. Si el beneficio neto de ser informal es mayor (menor), la gente optará por la informalidad (formalidad). Por tanto, a diferencia de los enfoques anteriores, este enfoque institucionalista considera la informalidad como resultado de una decisión voluntaria”.

El IGV llamado en otros países, Impuesto al Valor Agregado (IVA ), es considerado un impuesto indirecto ya que quien soporta la carga económica del mismo, no es el emprendedor de bienes o servicios, sino el Cliente (comprador o consumidor final) que 
es el Obligado.

El origen del IGV , en nuestro país, se remonta al Impuesto que daña en absoluto el precio de venta de los Timbres Fiscales (Ley 9923). Seguido con el Decreto Ley 19620( 1973) se estableció que “ el Impuesto a los Bienes y Servicios (IBS) gravaban la venta de bienes a nivel fabricante o importador".

Con Decreto Legislativo 190 (1982) se implementó que "un impuesto general al consumo sea con una tasa general del 16\%".

Con entrada en vigencia de la nueva constitución Política del estado del 1993, El IGV quedo regulado por el D. Leg. N.775 ley del Impuesto General a las Ventas e Impuesto Selectivo al Consumo, el cual está vigente hasta hoy, pero con diversas modificaciones a través de Leyes y Decretos Legislativos, que originaron el Decreto Supremo N. 055-99-EF, texto unico ordenado de la ley del impuesto general a las ventas Luego, en Agosto 2003 mediante la Ley 28033, se incrementó la tasa de IGV en $1 \%$; pasando de $16 \%$ a $17 \%$, resultando un total del $19 \%$ considerando el $2 \%$ de Impuesto de Promoción Municipal. Actualmente la tasa del IGV es de $18 \%$, está compuesta por una tasa de impuesto general al consumo del 16\% y la del Impuesto de Promoción $\mathrm{Mu}$ nicipal de $2 \%$. Tasa aplicable desde el 01.03.2011.Con lo que recauda el IGV se financia una buena parte del presupuesto nacional de la república, con lo que el estado puede brindar a toda la población servicios de Salud, Educación, infraestructura, seguridad, etc. (Según Información de la SUNAT2015, ingresos del Gobierno Central consolidado 1998-2015), "El impuesto con Mayor participación en la recaudación tributaria total, es el IGV, cuyo monto recaudado en el año 2014 fue de 55,613.40 millones de soles, representando el $52.78 \%$ del total de Ingresos Tributarios, monto que supera ampliamente lo recaudado por otros impuestos".

El Impuesto General a las Ventas, en el Perú es uno de los mas elevados en comparación con otros ,teniendo como paises encimade el a :Uruguay (22\%), Argentina (21\%), Chile (19\%). Los países que están por debajo de nuestra cifra son: Brasil (17\%), México y Colombia (16\%), Paraguay (15\%), Ecuador y Venezuela (12\%), Bolivia (13\%) y Panamá (7\%).

Según el Censo Inegi (2009) "El número de microempresas en el Perú alcanza a 4877,070 y el de pequeñas empresas a 214,956 . Por otro lado, el número de medianas y grandes empresas formales asciende a 52,030".

Según Produce (2015) "las MYPES brindan empleo a más de $80 \%$ de la población económicamente activa (PEA) y generan cerca de 45 por ciento del producto bruto interno (PBI). Constituyen, pues, el principal motor de desarrollo del Perú, su importancia se basa en que:

- Proporcionan abundantes puestos de trabajo. Reducen la pobreza por medio de actividades de generación de ingresos. Incentivan el espíritu empresarial y el carácter emprendedor de la población.• Son la principal fuente de desarrollo del sector privado. Mejoran la distribución del ingreso. Contribuyen al ingreso nacional y al crecimiento económico.

La constitución de una MYPE resulta socialmente deseable, por la inversión que realiza en la localidad donde se instala. Esta inversión tiene, además, tres elementos adicionales
- En primer lugar, Generación de empleos directos (es decir dentro de la misma empresa) y empleos indirectos que se generan gracias a la presencia de ésta, como por ejemplo, gracias a negocios como restaurantes, puestos de periódicos, venta de artesanías, construcciones metálicas, entre otros.

- En segundo lugar, permite a través de la aplicación de la oferta, que se ofrezcan mejores productos a menores precios, es decir un mercado más competitivo.

- En tercer lugar, hace posible que las personas tengan una actividad que les permita asumir retos y obtener logros, es decir desarrollarse a nivel personal-profesional.

(F. Villarán, 2007) dice: "En nuestro país, la situación actual de la micro, pequeña y mediana empresa es las siguiente: Representa el $99.5 \%$ de las empresas existentes, el $84 \%$ del empleo, el 64\% del PBI (solo las MYPE -sin medianas- y los independientes contribuyen con el $47 \%$ ), y el $2 \%$ de las exportaciones. Son un total de 2,5 millones de pequeñas empresas que dan empleo a 7,6 millones de personas (en esta cifra no se incluye el autoempleo), distribuidos: $57 \%$ en el ámbito urbano y $43 \%$ en el ámbito rural (referidos a la PEA). El 74\% de las MYPE (micro y pequeñas) son informales, aunque en la medida que crecen se va incrementando el grado de formalización (solo el $37 \%$ de las pequeñas son informales). Las pequeñas empresas son bastante descentralizadas, su PEA ocupada se distribuye así: $29,1 \%$ en la macro región norte, $23,6 \%$ en la macro región sur, $16 \%$ en la macro región centro, $7 \%$ en la macro región oriente, y solo $24,4 \%$ en Lima Metropolitana. Las actividades más importantes (en PEA ocupada) 
son: agricultura con $52,4 \%$, servicios con $18,3 \%$, comercio con $15,6 \%$, industria con $8,9 \%$ y construcción con 4,1\%”. (Fernando Villarán, 2007, El Mundo de la Pequeña Empresa, Pág. $11)$.

La informalidad, (Miranda, Sonia; 2016, PAG. 68-70), dice: "es el conjunto de unidades económicas que no cumplen con las regulaciones e impuestos,(...); las actividades INFORMALES se dividen en tres: 1) Evasión Tributaria pura, 2) Economía Irregular y 3) Actividades Ilegales.

La Evasión Tributaria pura, se produce cuando los individuos no reportan todos los ingresos generados a partir de actividades desempeñadas en negocios registrados y contabilizados en las estadísticas nacionales.

La Economía Irregular, comprende la producción de bienes y servicios legales en pequeños establecimientos que no son registrados.

Las Actividades Ilegales, abarca toda la producción y distribución ilegal de bienes y servicios". (Miranda Avalos, Sonia; pag.68-70; 2016)

Elsa Choy (2010), Revista Quipukamayoc, vol.17, pág., 128), afirma que: "En el Perú, las MYPES representan el 98,3\% del total de empresas existentes (94,4\% son micro y $3,9 \%$ son pequeñas), pero el $74 \%$ de ellas opera en la informalidad, La gran mayoría de las MYPE informales se ubican fuera de Lima, generan empleo de mala calidad (trabajadores familiares generalmente no remunerados) con ingresos inferiores a los alcanzados en las empresas formales similares.Las MYPE, además, aportan aproximadamente el $47 \%$ del PBI del país y son las mayores generadoras de empleo en la economía, aunque en la mayoría de los casos se trata de empleo informal.

En relación a la Presión Tributaria, la alta carga tributaria es en parte el poco interés de muchas MYPES informales en el Perú de pasar a la formalidad. La MYPE es informal simplemente porque no entiende ni cree en las ventajas de formalizarse”. (Choy, 2010)

Algunas causas de la expansión del Sector Informal son: Alto costo de la Formalización, crédito escaso para pequeñas empresas, desconocimiento del Marco legal ,fiscalizaciones establecidas por Ley ,la Pobreza es uno de los Factores del crecimiento de la economía informal ,poca Cultura tributaria, la migración de personas del campo a la ciudad ,el personal con poca capacitación y educación profesional y/o técnica, políticas y decisiones con falta de criterio ,administración Empírica y administración Tributaria poco eficiente.

El objetivo de la presente investigación es explicar la Existencia de influencia de la Dimensión de la Informalidad de las MYPES en la Recaudación del IGV, durante el periodo 2010-2014, y por qué la Informalidad de las Mypes fue un factor que influencio en la Recaudación del IGV. Periodo 2010-2014.

\section{MATERIAL Y MÉTODOS}

El estudio realizado es Descriptivo y Explicativo; ya que consiste en plantear lo más relevante de un hecho o situación concreta, y explicativo porque no solo se describe el problema sino que también busco explicar las causas que originaron la situación que se analiza en este trabajo de investigación.

Se analizó la evolución de la informalidad en las Mypes peruanas y como esta afecta la recaudación fiscal. Asi- mismo de describen los resultados de la influencia de las Mypes informales en la economía.

El estudio corresponde a un diseño no experimental - Transaccional - Correlaciona: no experimenta, porque no se realizan pruebas donde las variables de investigación son manipuladas; Transaccional, porque el objeto de estudio se analizara en un determinado periodo de tiempo; Correlacional, por que plantea la existencia de relación de tipo estadístico entre variables cuantitativas. El enfoque es cuantitativo, pues se prueba a través de mediciones estadísticas; El Objeto de estudio es la Informalidad de las Mypes Peruanas.

\section{RESULTADOS}

\section{Estimación total de Mypes en el Perú}

Según Produce (2015) en su publicación, las Mipymes en Cifras 2013,y las Mipymes en cifras 2014 (Pág. 130 133), realiza una estimación del número total de Mypes en el Perú entre los años 2010-2014, así como determina aproximadamente el número de las Mypes informales; dice así: "Debido a la carencia de información y a la elevada informalidad que existe en el estrato de Micro y Pequeña Empresa, es complicado obtener cifras precisas del tamaño del sector. Ante esa situación, se hace uso del módulo de Empleo e Ingresos - módulo cinco- de la ENAHO para aproximar el tamaño total del sector. Para ello existen dos metodologías." (p.74-76)

a) La del Rango de Trabajadores.

b) La del Número de Conductores.

En este documento se utiliza el método de conductores de empresas debido a que en este caso es el propio conductor quien reporta la existencia del 
negocio o empresa (En la estimación se considera únicamente el reporte de la ocupación principal)”.

Tabla 1. Estimación del total de Mypes 2010-2014 de acuerdo al número de conductores.

Fuente: INEI - Encuesta Nacional de Hogares metodología actualizada - Elaboración propia.

\begin{tabular}{lccccc}
\multicolumn{1}{c}{ TAMAÑO } & $\mathbf{2 0 1 0}$ & $\mathbf{2 0 1 1}$ & $\mathbf{2 0 1 2}$ & $\mathbf{2 0 1 3}$ & $\mathbf{2 0 1 4}$ \\
MICRO EMPRESAS & 3162344 & 3127128 & 3173065 & 3024891 & 3600543 \\
\hline PEQUEÑS EMPRESAS & 41593 & 42682 & 45644 & 35570 & 37177 \\
\hline TOTAL MYPES & 3203937 & 3169810 & 3218709 & 3060461 & 3637720 \\
\hline
\end{tabular}

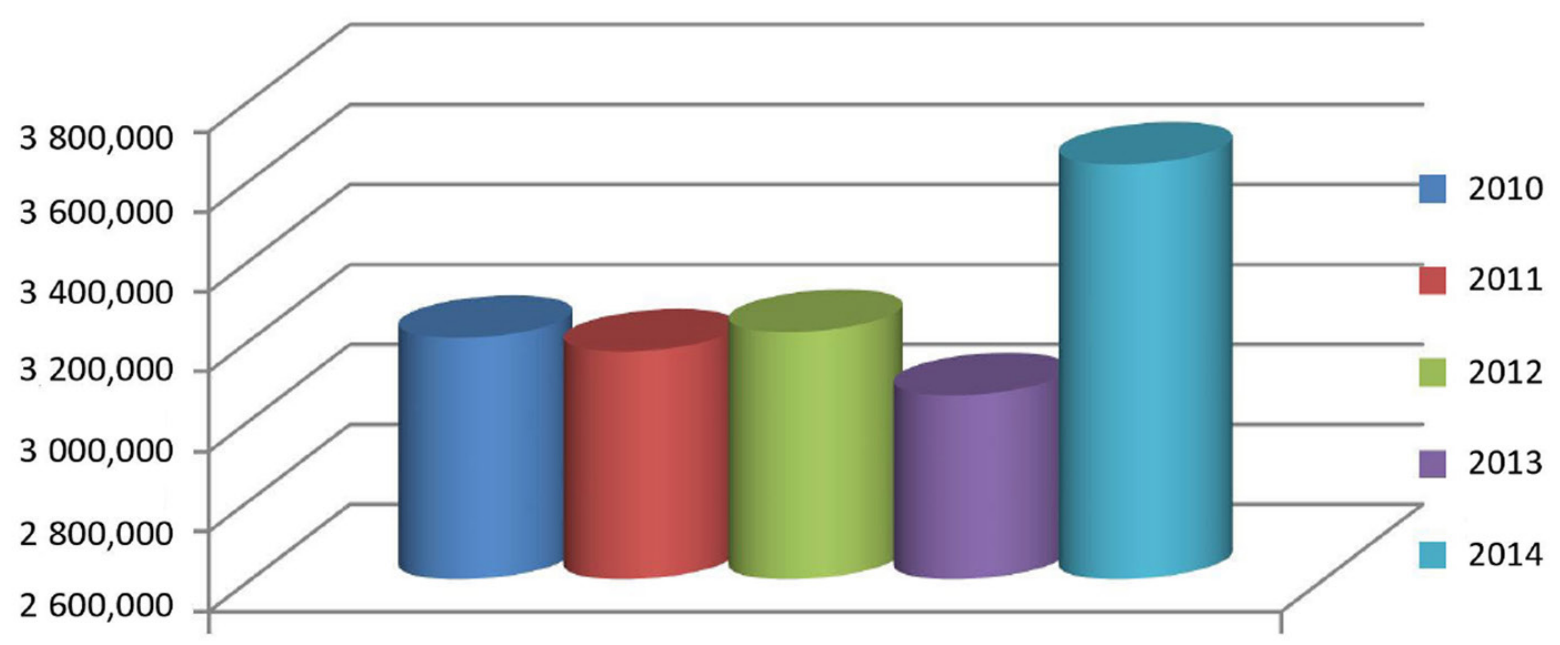

Figura 1. Total de Mypes.

Fuente: INEI.

\section{Interpretación (Figura 1 y 2 )}

Estimación del Número de Mypes Formales, según el Ministerio de la Producción, en su publicación, las Mipymes en Cifras 2013, (Pág. 74-76) y las Mipymes en cifras 2014 (Pág. 130-133), realiza una estimación del número total de Mypes en el Perú entre los años 2010-2014, así como determina aproximadamente el número de las Mypes informales; dice así:

Según Diaz (2014) sostiene que "existe una amplia heterogeneidad de dimensiones de la informalidad, lo que dificulta encontrar el número de empresas formales e informales de una manera única sintetiza en dos dimensiones la informalidad: informalidad laboral e informalidad tributaria. En el caso de la informalidad laboral, distingue varios criterios (rasgos) para identificar las obligaciones propias de una relación laboral como el acceso a un seguro de salud, una pensión de jubilación, a gratificaciones y a un contrato de trabajo. Asimismo, en el caso de la informalidad tributaria, también distingue criterios, como la tenencia de RUC de la empresa, si la empresa tiene un sistema de contabilidad, y si la empresa está registrada como persona jurídica.
En este documento se utiliza la tenencia de RUC como el criterio tributario para medir la informalidad".

Sunat sostiene que "para calcular el número de micro y pequeñas empresas formales, utilizamos el Registro Único del Contribuyente, proporcionado por la Sunat. Según el criterio tributario, el número de mypes informales se obtiene de la diferencia entre el total de micro y pequeñas empresas estimado con la información de ENA$\mathrm{HO}$, y las registradas en el padrón de RUC de las Sunat". 
Tabla 2. Micro y pequeñas empresas (MYPES).

Fuente: Sunat, Registro Unico del Contribuyente 2009-2013 - Elaboración propia.

\begin{tabular}{lccrrr}
\multicolumn{1}{c}{ TAMAÑO } & $\mathbf{2 0 1 0}$ & $\mathbf{2 0 1 1}$ & $\mathbf{2 0 1 2}$ & $\mathbf{2 0 1 3}$ & $\mathbf{2 0 1 4}$ \\
MICRO EMPRESAS & 1179275 & 1267060 & 1321992 & 1496320 & 1571121 \\
\hline PEQUEÑAS EMPRESAS & 20072 & 22047 & 23398 & 22149 & 25940 \\
\hline TOTAL MYPES & 1199347 & 1289107 & 1345390 & 1518469 & 1597061 \\
\hline
\end{tabular}

Nota: El estrato empresarial es determinado en base al rango de trabajadores: Microempresa hasta 10 trabajadores y pequeñas empresas de 11 a100 trabajadores.

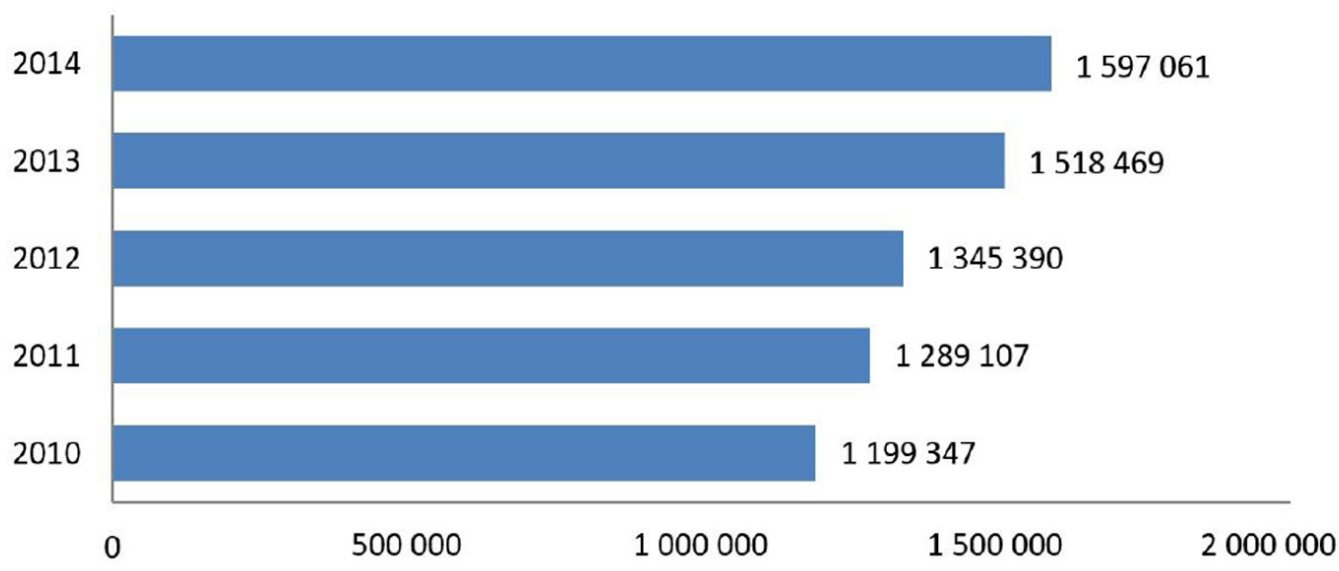

Figura 2. Evolución de la cantidad de MYPES formales.

Fuente: Sunat, Registro Unico del Contribuyente 2009-2013, 2010-2014.

Estimación del Número de Mypes Informales

En la figura 2 se muestran los resultados de la estimación de Mypes informales para el periodo 2010-2014. A partir de estas estimaciones se puede calcular la evolución de la participación de las micro y pequeñas empresas formales e informales desde el punto de vista tributario. Dado que el aumento del número de mypes for- males ha sido mucho más rápido que el número total de mypes, el porcentaje de micro y pequeñas empresas formales, desde el punto de vista tributario, muestra una tendencia continuamente creciente.

Tabla 3. Estimación del Numero de MYPES informales, período 2010-2014.

Fuente: Sunat,INEI -Encuesta Nacional de Hogares - Elaboración propia.

\begin{tabular}{|c|c|c|c|c|c|}
\hline AÑO & $\begin{array}{l}\text { NUMERO ESTIMADO } \\
\text { DE MYPES }\end{array}$ & MYPES FORMALES & $\begin{array}{l}\text { MYPES INFORMALES } \\
\text { (ESTIMACION POR } \\
\text { DIFERENCIA) }\end{array}$ & \% DE MYPES FORMALES & $\begin{array}{l}\text { \% DE MYPES } \\
\text { INFORMALES }\end{array}$ \\
\hline 2010 & 3203937 & 1199347 & 2004590 & $37,40 \%$ & $62,60 \%$ \\
\hline 2011 & 3169810 & 1289107 & 1880703 & $40,70 \%$ & $59,30 \%$ \\
\hline 2012 & 3218709 & 1345390 & 1873319 & $41,80 \%$ & $58,20 \%$ \\
\hline 2013 & 3060461 & 1518469 & 1541992 & $49,60 \%$ & $50,40 \%$ \\
\hline 2014 & 3637720 & 1597061 & 2040659 & $43,90 \%$ & $56,10 \%$ \\
\hline
\end{tabular}

Nota: El número total de mypes se estima con la información dada en la ENAHO. 


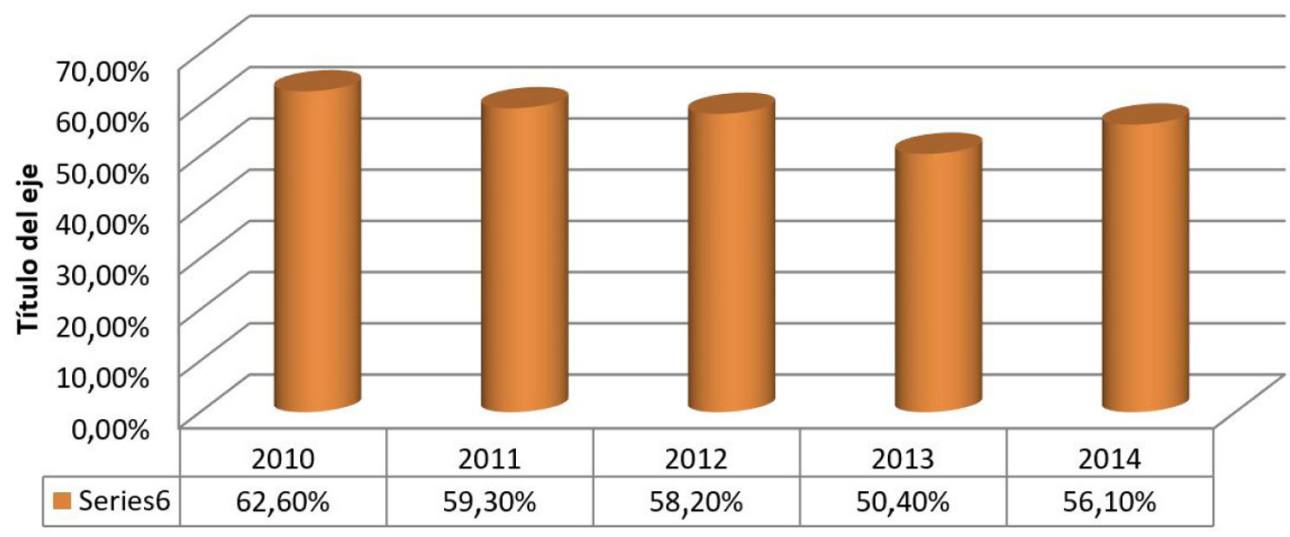

Figura 3. Evolución de la informalidad en el Peru 2010-2014.

Fuente: Sunat, INEI -Encuesta Nacional de Hogares - Elaboración propia.

Estimación de la Influencia de la Informalidad en las pymes, en la Recaudación del IGV

De acuerdo a la información Obtenida del Ministerio de la Producción, en sus publicaciones las Mipymes en cifras 2013 y 2014, de la encuesta Nacional de Hogares, ENAHO utilizando el método de conductores, el Registro único de Contribuyentes 2009-2013 y 2010-2014, proporcionado por la Sunat, a partir de esta información y estimaciones se ha calculado de manera aproximativa la Influencia de las mypes Informales en la economía nacional, específicamente en la recaudación del IGV y su influencia en el total de la Recaudación Tributaria.

Tabla 4. Estimación de la Influencia de las MYPES informales en la recaudación del IGV. Fuente: Sunat,INEI -Encuesta Nacional de Hogares - Elaboración propia.

\begin{tabular}{lcccc}
\multicolumn{1}{c}{ CATEGORIA } & TOTAL MYPES & $\begin{array}{c}\text { \% DEL TOTAL DE } \\
\text { MYPES }\end{array}$ & $\begin{array}{c}\text { RECAUDACION IGV } \\
\text { MYPES }\end{array}$ & $\begin{array}{c}\text { \% DEL TOTAL INGRESOS } \\
\text { TRIBUTARIOS }\end{array}$ \\
\hline MYPES FORMALES & 1391085 & $42,68 \%$ & 43636,48 & $53,44 \%$ \\
\hline $\begin{array}{l}\text { MYPES INFORMA- } \\
\text { LES }\end{array}$ & 1868253 & $57,32 \%$ & 58604,60 & $71,77 \%$ \\
\hline TOTALES & 3259338 & $100,00 \%$ & 102241,08 & \\
\hline
\end{tabular}

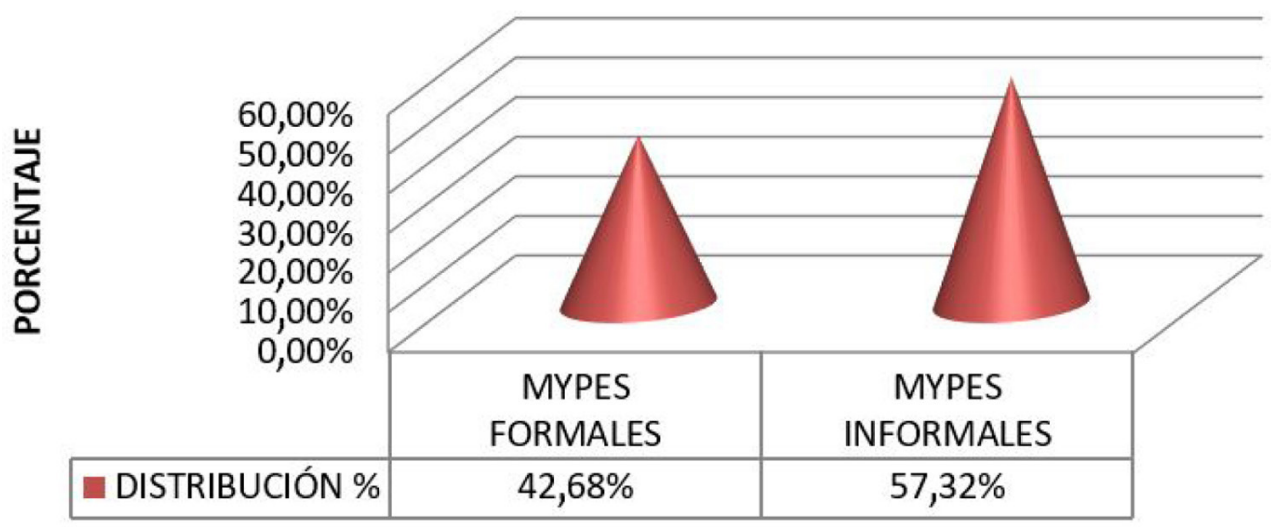

Figura 4. Distribución estimada de las MYPES en el Perú.

Fuente: Sunat,INEI - Encuesta Nacional de Hogares - Elaboración propia. 
La Información contenida en la figura 5 , se obtiene del promedio de los años 2010 al 2014, de los datos contenido en las figuras 1, 2, 3, 4. De esta forma se muestran los resultados de la estimación de la cantidad de Mypes Formales e Informales y el porcentaje que estas representarían del Total para el periodo 2010-2014. A partir de estas estimaciones se puede calcular la evolución de la participación de las Mypes Formales e Informales desde el punto de vista Tributario. En este sentido en los años de estudio de esta investigación el promedio de MYPES Formales es de 1, 391,085 y cuya participación seria del $42.68 \%$ del total de MYPES; así mismo el Promedio de MYPES Informales seria de 1, 868,253 con una participación del $57.32 \%$ con relación al total de MYPES estimadas. Así mismo del promedio de MYPES
Formales cuya participación seria del 42.68\% del total de MYPES se obtiene una recaudación promedio en base a datos oficiales de 43,636.48 (Millones de soles); así mismo el Promedio de MYPES Informales tendría con una participación del $57.32 \%$ con relación al total de MYPES estimadas dejando de aportar al fisco un monto estimado de 58,604.60( Millones de soles ).

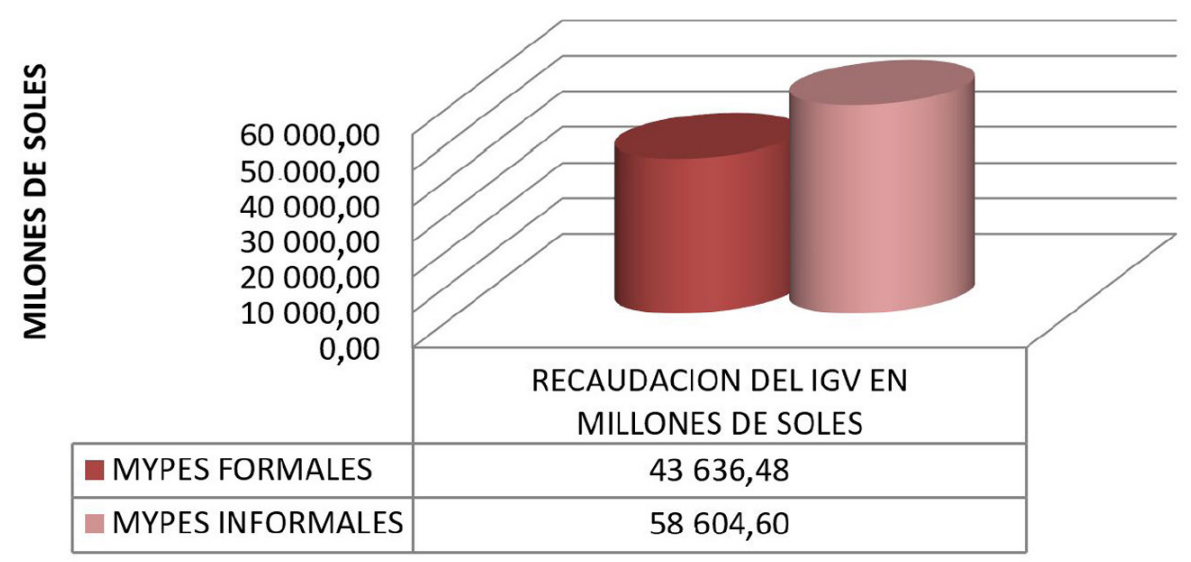

Figura 5. Monto estimado de ingv no pagado por las Mypes informales.

Fuente: Sunat, INEI -Encuesta Nacional de Hogares - Elaboración propia.

Así mismo, del total de MYPES, el $42.68 \%$ promedio, corresponden a las MYPES Formales que tendrían una participación en el total de Ingresos
Tributarios equivalente al 53.44\%.

Del mismo modo, del total de MYPES, el $57.32 \%$ promedio, corresponderían a las MYPES Informales que tendrían una participación en el total de Ingresos Tributarios equivalente al $71.77 \%$.

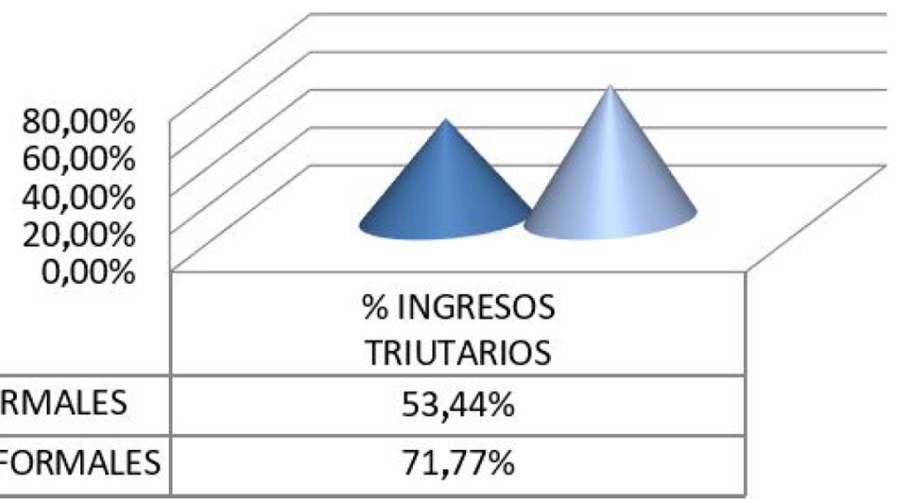

Figura 6. \% Total de Ingreso Tributarios.

Fuente: Sunat, INEI -Encuesta Nacional de Hogares - Elaboración propia. 


\section{DISCUSIÓN}

Los Altos índices de desempleo y pobreza que afecta nuestro País, influye en nuestro desarrollo económico y social, en donde a la escases de empleo surge como alternativa, el empleo Independiente, a través de la creación de micro y pequeñas empresas(MYPES).

Okpara y Wynn (2007) afirman que: "los pequeños negocios son considerados como la fuerza impulsora del crecimiento económico, la generación de empleo y la reducción de la pobreza en los países en desarrollo"; pero hay un fenómeno que está estrechamente vinculado a las mypes: la informalidad que, según la sunat representa para nuestra economía el $57,9 \%$ del PBI y es el principal obstáculo para lograr la ampliación de la base tributaria.

Además muchas Mypes no reúnen las condiciones para ingresar, desarrollarse y crecer en un mercado específico. También, la alta tasa de informalidad presente en las Mypes, afecta significativamente la recaudación fiscal, específicamente la recaudación del IGV, impidiendo el crecimiento de nuestra economía.

Para afrontarla, es necesario identificar donde y en qué cantidad existen, las causas que la originan y que hace se mantengan en ella, y cuál es su influencia en los ingresos tributarios del Gobierno Central, correspondientes al pago del IGV.

Mediante el análisis estadístico de los datos recopilados correspondiente al periodo de investigación se pudo determinar qué, el promedio de MYPES Formales es de 1391085 y cuya participación seria del $42,68 \%$ del total de MYPES; así mismo el Promedio de MYPES Informales seria de 1,868,253 con una participación del $57,32 \%$ con relación al total de MYPES estimadas.

\section{REFERENCIAS BIBLIOGRAFÍCAS}

Choy, E. (2011). La Informalidad en los Sectores Económicos y la Evasión tributaria en el Perú. Quipukamayoc. 18(35).

Gomero, N. (2015). Concentración de las Mypes y su Impacto en el Crecimiento Económico. Quipukamayoc, $23(43)$.
Ministerio de la Producción. (2011). Dirección General de Estudios Económicos, Evaluación y Competitividad Territorial Estadísticas de la Micro y Pequeña Empresa. Lima: Registro Oficial,

Ministerio de la Producción. (2014). Dirección General de Estudios Económicos, Evaluación y Competitividad Territorial Las Mipymes en Cifras, Registro Oficial,Lima.

Ministerio de la Producción, (2015) Dirección General de Estudios Económicos, Evaluación y Competitividad Territorial.Lima: Las Mipymes en Cifras, Registro Oficial,

Martha, C. (2012). Mujeres en empleo informal: Globalizado y Organizado; Lambayeque.

Villarán,F. (2007) El mundo de la Pequeña Empresa en el Perú. COPEME-CONFIEP- MINCETUR, lima

Villarán; F,. (1998). Riqueza Popular, Pasión y gloria de la Pequeña Empresa, Congreso del Perú. 